\title{
Toxoplasmosis intramedular en una paciente con coinfección por VIH y tuberculosis
}

\author{
Giancarlo Pérez-Lazo, Raúl Castillo-Córdova y Julio Maquera-Afaray
}

\section{Intramedullary toxoplasmosis in HIV-tuberculosis co-infected patient}

The most common clinical presentation of Toxoplasma gondii in HIV patients is encephalitis; however, the intramedullary involvement has been reported in a few cases. We report a case of intramedullary toxoplasmosis in a female patient diagnosed with HIV/tuberculosis co-infection, and history of poor adherence to antiretroviral therapy. The patient developed subacute paraparesis with compromise of sensory function and urinary sphincter. The nuclear magnetic resonance evaluation showed a single intramedullary ring-enhanced lesion at the T-8 level which was solved after an anti-Toxoplasma therapy with trimethoprim/ sulfamethoxazole.

Key words: Acquired immunodeficiency syndrome, myelitis, spinal cord lesion, toxoplasmosis.

Palabras clave: Síndrome de inmunodeficiencia adquirida, mielitis, lesión cordón espinal, toxoplasmosis.

\section{Introducción}

La toxoplasmosis, ocasionada por el protozoo Toxoplasma gondii, es una infección parasitaria con distribución global y que prevalece como la infección neurológica oportunista más frecuente en personas con infección por VIH/SIDA ${ }^{1,2}$.

En Sudamérica, continúa siendo una infección frecuente del sistema nervioso central (SNC), causando encefalitis o masa cerebral ${ }^{1}$. Esta afección se encontró en 6,7\% de los pacientes con VIH tratados en un hospital peruano ${ }^{3}$. Sin embargo, esporádicamente se han descrito casos de toxoplasmosis con compromiso de médula espinal, predominantemente en pacientes inmunocomprometidos, por deficiencia inmunológica de las células T CD4+ asociado a infección por VIH o leucemia/linfoma de células $\mathrm{T}^{4}$.

Comunicamos un caso de toxoplasmosis intramedular en una paciente con coinfección por VIH y tuberculosis, con respuesta favorable, con evidencia imagenológica, a una prueba terapéutica con cotrimoxazol.

\section{Caso clínico}

Paciente mujer de 33 años con historia de 10 años de infección por VIH, con antecedente de una tuberculosis pulmonar al momento del diagnóstico. Recibió terapia anti-retroviral (TARV) con buena adherencia por ocho años, con respuesta inmunológica y virológica favorable, la que abandonó, según refirió, por mejoría clínica. A los dos años del abandono, fue diagnosticada nuevamente de tuberculosis, con compromiso ganglionar y multirresis- tencia a fármacos antituberculosos. Había recibido terapia con un régimen individualizado (amikacina, pirazinamida, cicloserina, etionamida y levofloxacina) por siete meses, con reinicio a las cuatro semanas de la TARV previa (tenofovir, lamivudina, efavirenz). Sin embargo, finalmente había abandonado ambos esquemas por intolerancia a la medicación.

Además, la paciente tenía el antecedente de haber sido tratada en forma empírica por una encefalitis por Toxoplasma con cotrimoxazol $(5 \mathrm{mg} / \mathrm{kg} /$ día de trimetoprim, fraccionado cada $12 \mathrm{~h}$ ) por seis semanas, con respuesta favorable clínica y por control de imágenes por resonancia magnética (RM) (Figura 1a, 1b). Al alta, había reiniciado el tratamiento antituberculoso, y se había continuado con cotrimoxazol, con dosis de mantención $(160 / 800 \mathrm{mg}$, cada $24 \mathrm{~h}$ ); sin adherencia regular.

Tres meses después reingresó por Servicio de Emergencias con un cuadro neurológico, de aproximadamente una semana de evolución, caracterizado por paraparesia progresiva en miembros inferiores, retención urinaria y compromiso sensitivo en nivel T8. Se realizó RM de columna y médula espinal (Figura 2) revelando la presencia de una imagen intramedular captadora de contraste en anillo $(0,9 \mathrm{~cm} \times 2,6 \mathrm{~cm})$ con edema perilesional, a nivel de T8.

El estudio de líquido cefalorraquídeo (LCR) mostró 20 células a predominio linfomononuclear (90\%), hiperproteinorraquia $(247 \mathrm{mg} / \mathrm{dL})$, glucorraquia y prueba de ADA (adenosina deaminasa) dentro de límites normales. Los exámenes microbiológicos en LCR para bacterias (tinción Gram y cultivo), hongos (tinta china y antígeno para Cryptococcus) y micobacterias (baciloscopias y Ge-
Hospital Guillermo Almenara, Lima, Perú.

Servicio de Medicina InternaInfectología (GPL, RCC, JMA).

Recibido: 8 de mayo de 2016 Aceptado: 9 de diciembre de 2016

Correspondencia a: Giancarlo Pérez-Lazo giancarlo.perez@unmsm.edu.pe 



Figura 1 (1a, 1b). RM cerebral corte sagital y coronal con gadolinio. Evidencia múltiples lesiones nodulares con captación periférica central y nodular, con algunas áreas irregulares, en ambos hemisferios cerebrales, hemisferio cerebeloso derecho, región temporal derecha, cabeza de núcleos caudado derecho, tálamo izquierdo, y lóbulo parietal derecho, que se asocian a edema vasogénico leve en parénquima circundante. Lesiones corresponden a toxoplasmosis cerebral, 3 meses antes del episodio de la afectación intramedular.

neXpert MTB/RIF) fueron negativos. No se realizó RPC para Toxoplasma en LCR, y las cargas virales para CMV y VEB séricas y en LCR fueron indetectables. El recuento de células T CD4+ fue 11 céls $/ \mathrm{mm}^{3}$, con carga viral VIH de 548.800 copias ARN/ml; los títulos séricos de IgG para Toxoplasma fueron positivos en 4,37 $(<0,8=$ negativo $)$ obtenidos mediante inmunoanálisis quimioluminiscente de partículas (CMIA).

Se reinició terapia empírica, debido a que no se pudo realizar una biopsia de la lesión intramedular, con cotrimoxazol i.v. ( $5 \mathrm{mg} / \mathrm{kg}$ de trimetoprim, dos veces al día), durante cuatro semanas asociado a dexametasona i.v. $8 \mathrm{mg}$, fraccionado cada $12 \mathrm{~h}$, por siete días. Posteriormente continuó dos semanas con cotrimoxazol a la misma dosis por vía oral, y reducción semanal progresiva de corticoesteroides con prednisona, hasta completar seis semanas. Tuvo control imagenológico cuatro semanas después del tratamiento, evidenciándose mejoría de lesión intramedular, con evidente disminución de tamaño y menor realce al contraste (Figura 3). Clínicamente a la semana de tratamiento empírico, comenzó a evidenciarse una mejoría de la sensibilidad discriminatoria y la fuerza muscular; sin embargo, persistió con compromiso del esfinter urinario (retención urinaria). Ante la mejoría inicial, se decidió continuar con tratamiento para toxoplasmosis (durante seis semanas), y terapia de rehabilitación física. Previo al alta se reinició la TARV con mismo esquema recibido previamente. Finalmente, la paciente continuó la terapia antituberculosa de manera regular y con dosis de mantención con cotrimoxazol 160/800 mg, fraccionado cada $12 \mathrm{~h}$.

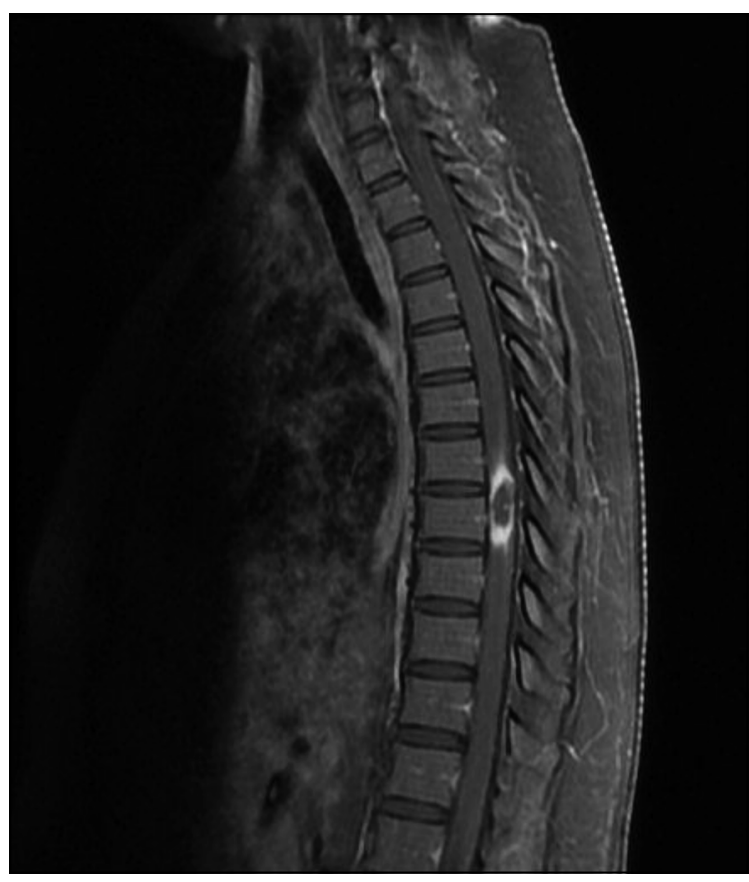

Figura 2. RM toraco-lumbar, corte sagital T1 (con gadolinio), se evidencia lesión única con captación de contraste periférico en anillo $(0,9 \mathrm{~cm} \times 2,6 \mathrm{~cm})$ a nivel de T8.

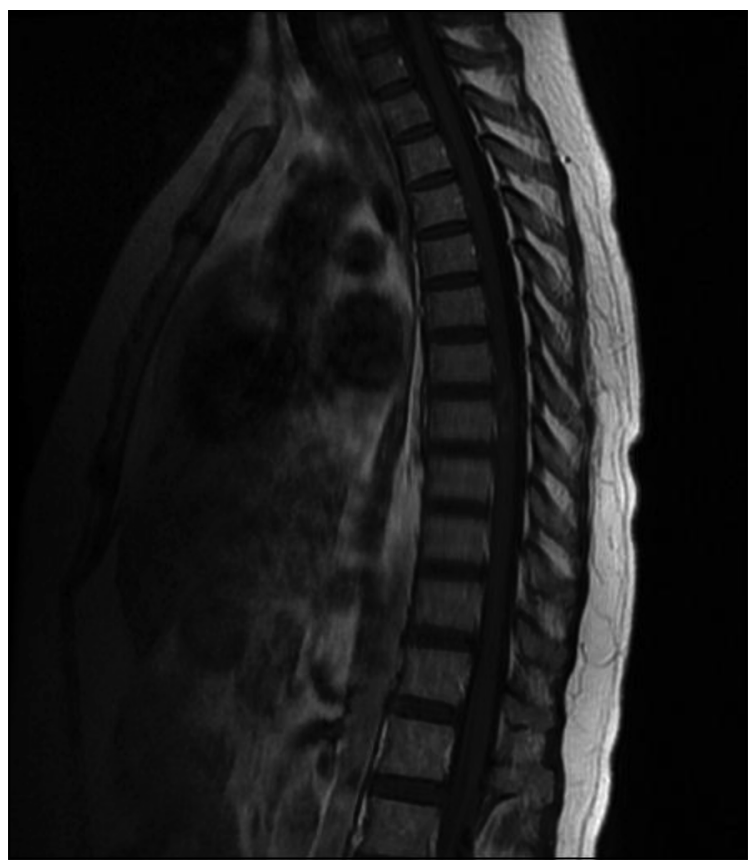

Figura 3. RM toraco-lumbar, corte sagital en T1 (con gadolinio), se evidencia tras 4 semanas de tratamiento con cotrimoxazol, disminución del tamaño de la lesión y menor captación de contraste periférico a nivel de T8. Se aprecia leve persistencia de compromiso intramedular dorsal asociado a cambios de aspectos mielomalácicos. 


\section{Discusión}

Hasta la actualidad, han sido reportados aproximadamente 25 casos de toxoplasmosis intramedular asociada a infección por VIH, diagnosticados por histopatología (biopsia o autopsia) o por respuesta a una prueba terapéutica. Nuestro caso correspondió a una paciente de 33 años de edad y sexo femenino, con diagnóstico de coinfección por VIH y tuberculosis, y mala adherencia a la TARV; cabe mencionar que en la mayoría de los pacientes documentados con toxoplasmosis intramedular, $86 \%$ fueron varones y la edad media fue de 41 años $^{5}$.

Las manifestaciones neurológicas de la toxoplasmosis intramedular descritas son principalmente la pérdida de la función motora con compromiso de las extremidades inferiores, disfunción de esfínteres, y alteración sensitiva, características clínicas concordantes con el caso ${ }^{6}$. Además, la RM debería considerarse como el examen imagenológico de elección ${ }^{7}$, ya que permite evidenciar la lesión intramedular con un realce en anillo como el hallazgo más frecuente, comprometiendo el segmento torácico en la mayoría de casos. El compromiso simultáneo encefálico-medular por Toxoplasma se ha presentado en la mitad de los casos descritos ${ }^{5}$. Por otro lado, aunque el estudio de LCR resulta inespecífico en los casos de toxoplasmosis intramedular, con presencia de pleocitosis e hiperproteinorraquia, debería efectuarse como parte del estudio diferencial de otras patologías. La biología molecular cuando está disponible, como la RPC para Toxoplasma, debido a su elevada especificidad y sensibilidad es una herramienta diagnóstica importante a considerar ${ }^{8}$. Del mismo modo, el título de anticuerpos IgG para Toxoplasma es útil en el diagnóstico, pero esto no es absoluto ya que la serología en pacientes intensamente inmunocomprometidos, especialmente en pacientes con SIDA, puede no mostrar elevación de títulos e incluso ser negativa. Por lo tanto, la seronegatividad no excluye el diagnóstico ${ }^{9,10}$. En los casos de toxoplasmosis intramedular reportados, incluyendo el nuestro, la serología resultó positiva. El diagnóstico definitivo de toxoplasmosis por la demostración del microorganismo en el estudio histopatológico está reservado para aquellos pacientes en quienes no se evidencia respuesta a la terapia antiparasitaria9.

En relación al tratamiento de la toxoplasmosis intramedular, no existen estudios que avalen un fármaco determinado; sin embargo, la mayoría de los casos fueron tratados con buenos resultados con esquemas para encefalitis por Toxoplasma ${ }^{5,11}$; siendo, pirimetamina-sulfadiazina (con ácido folínico) la terapia utilizada en la mayoría de los reportes. En la seguridad social de Perú, no disponemos de pirimetamina, considerada el régimen de elección, por lo que nuestra paciente fue tratada con cotrimoxazol ( $5 \mathrm{mg} / \mathrm{kg}$ de trimetoprim, fraccionado cada $12 \mathrm{~h}$ ), según las recomendaciones internacionales ${ }^{9} \mathrm{y}$ basado en que no se ha demostrado diferencia entre ambos esquemas de tratamiento en encefalitis por Toxoplasma ${ }^{12,13}$. Aquellos pacientes con toxoplasmosis intramedular que no recibieron ningún tipo de tratamiento antiparasitario fallecieron ${ }^{5}$. El uso de corticoesteroides asociados en encefalitis por Toxoplasma no está avalado, debido que su efecto podría enmascarar la presencia de un linfoma primario cerebral retardando el diagnóstico ${ }^{9}$. Respecto al uso de corticoesteroides en casos de localización intramedular, fueron utilizados en ocho casos documentados, cuatro de ellos con resolución completa, tres con respuesta parcial y un fallecido ${ }^{5}$. En nuestro caso, el tratamiento con corticoesteroides fue administrado durante seis semanas, asociado al tratamiento antiparasitario, con respuesta imagenológica pero con respuesta clínica incompleta, dejando secuelas neurológicas. Hace falta aún mayor evidencia que nos permita conocer la utilidad de los corticoesteroides en esta patología.

Por otro lado, ante la ausencia de respuesta o deterioro clínico después de dos semanas de tratamiento debe considerarse un diagnóstico alternativo9. Sin embargo, se necesita mayor evidencia que sostenga esta estrategia en la toxoplasmosis intramedular, así como ocurre en encefalitis, y determine de manera adecuada el seguimiento y valoración de estos pacientes. García-García demostró la reducción de lesiones tanto cerebrales como del cordón espinal después de 15 días de tratamiento para toxoplasmosis, similar a lo que ocurre en la encefalitis. No obstante, de la serie de casos sólo nueve de 26 tuvieron resolución completa de la enfermedad, siendo aún poco claro precisar si seis semanas de tratamiento agudo son suficientes antes de pasar a la fase de mantención como en la encefalitis por Toxoplasm $a^{5,9}$. En nuestro caso, nosotros optamos por seguir estas recomendaciones, con terapia de mantención luego de la sexta semana, considerando también la carga de píldoras al día que la paciente recibía y la mala adherencia previa (a la terapia antituberculosa, TARV y cotrimoxazol).

En relación al diagnóstico diferencial de lesiones medulares, existen diferentes causas infecciosas y neoplásicas de mielopatía en pacientes con infección por VIH/SIDA, con localización extradural, intraduralextramedular, e intramedular. Thurnher y cols. encontraron de un total de 55 pacientes con infección por VIH que presentaron manifestaciones neurológicas vinculadas con compromiso de cordón espinal, nueve tuvieron una enfermedad intramedular evidenciada por RM; de los cuales, la mayoría fueron por Toxoplasma, seguido por la mielopatía vacuolar, mielitis herpética y linfoma intramedular ${ }^{14}$.

No obstante, en el caso presentado, por el antecedente de tuberculosis, se consideró como principal diagnóstico diferencial una posible lesión medular secundaria a esta enfermedad, en el contexto de una paciente con adherencia irregular a la terapia. A pesar de que la incidencia de 
tuberculosis espinal representa sólo $1 \%$ de todos los casos de tuberculosis, usualmente afecta adultos jóvenes, con predominancia en varones, siendo la afectación torácica la más frecuente ${ }^{15}$.

\section{Conclusión}

Aunque la toxoplasmosis intramedular es una enfermedad infrecuente, debe plantearse oportunamente entre las alternativas diagnósticas en un paciente inmunocomprometido con síntomas y/o signos de mielopatía aguda o subaguda, en vista que es una enfermedad tratable, y cuyo pronóstico depende del diagnóstico y tratamiento temprano.

\section{Resumen}

El compromiso encefálico por Toxoplasma gondii en pacientes con VIH es la localización más frecuente, no obstante, la localización intramedular ha sido escasamente reportada. Comunicamos un caso de toxoplasmosis intramedular en una mujer con diagnóstico de coinfección por VIH y tuberculosis, con mala adherencia a la terapia antirretroviral, que desarrolló de forma subaguda un cuadro de paraparesia con compromiso sensitivo y de esfínteres. La resonancia magnética mostró una lesión única intramedular con captación de contraste periférico en anillo a nivel T-8, que se resolvió tras recibir tratamiento anti-toxoplasmosis con cotrimoxazol.

\section{Referencias bibliográficas}

1.- Tan I L, Smith B R, von Geldern G, Mateen F J, McArthur J C. HIV-associated opportunistic infections of the CNS. Lancet Neurol 2012; 11 : 605-17.

2.- Kodym P, Malý M, Beran O, Jilich D, Rozsypal H, Machala L, et al. Incidence, immunological and clinical characteristics of reactivation of latent Toxoplasma gondii infection in HIV-infected patients. Epidemiol Infect 2015; 143: 600-7.

3.- Cáceda R, Seas C, Echevarría J, Samalvides F, León Y, Gotuzzo E. Toxoplasmosis cerebral en pacientes con SIDA en el Hospital Nacional Cayetano Heredia entre 1989 y 1999. Rev Med Hered 2000; 11: 15-21.

4.- do Amaral L L, Nunes R H, da Rocha A J. Parasitic and rare spinal infections. Neuroimaging Clin N Am 2015; 25: 259-79.

5.- García-García C, Castillo-Álvarez F, AzconaGutiérrez J M, Herraiz M J, Ibarra V, Oteo J A. Spinal cord toxoplasmosis in human immunodeficiency virus. Infect Dis (Lond) $2015 ; 47: 277-82$
6.- Rodríguez C, Martínez E, Bolívar G. Sánchez $\mathrm{S}$, Carrascal E. Toxoplasmosis of the spinal cord in an immunocompromised patient: case report and review of the literature. Colomb Med 2013; 44: 232-5.

7.- Li Y-F, Li H-J, Zhang Q, Liu J-J, Wang W, Zhang Y-Y. MRI demonstrations of AIDS complicated by Toxoplasma gondii infection in cervical spinal cord with 3 cases reports. Radiol Infect Dis 2015; 2: 159-61.

8.- San Martín F J, Baptista T, Araujo C. Toxoplasmosis cerebral e medular: a propósito de um caso clínico. Medicina Interna 2008; 15 : 172-76.

9.- Guidelines for the Prevention and Treatment of Opportunistic Infections in HIV-Infected Adults and Adolescents. Department of Health and Human Services, 2015. Available at: https:// aidsinfo.nih.gov/guidelines/html/4/adultand-adolescent-oi-prevention-and-treatmentguidelines $/ 0$. Fecha de acceso: 20 de febrero de 2016.

10.- Derouin F, Leport C, Pueyo S, Morlat P, Letrillart B, Chene G, et al. Predictive value of Toxoplasma gondii antibody titres on the occurrence of toxoplasmic encephalitis in HIVinfected patients. ANRS 005/ACTG 154 Trial Group. AIDS 1996; 10: 1521-7.

11.- García-Gubern C, Fuentes C R, Colon-Rolon L, Masvidal D. Spinal cord toxoplasmosis as an unusual presentation of AIDS: case report and review of the literature. Int J Emerg Med 2010; 3: 439-42.

12.- Beraud G, Pierre-Francois S, Foltzer A, Abel S, Liautaud B, Smadja D, et al. Cotrimoxazole for treatment of cerebral toxoplasmosis: an observational cohort study during 1994-2006. Am J Trop Med Hyg 2009; 80: 583-7.

13.- Yan J, Huang B, Liu G, Wu B, Huang S, Zheng $\mathrm{H}$, et al. Meta-analysis of prevention and treatment of toxoplasmic encephalitis in HIVinfected patients. Acta Trop 2013; 127: 236-44.

14.- Thurnher M M, Post M J, Jinkins J R. MRI of infections and neoplasms of the spine and spinal cord in 55 patients with AIDS. Neuroradiology 2000; 42: 551-63.

15.- Ramdurg S R, Gupta D K, Suri A, Sharma B S, Mahapatra A K. Spinal intramedullary tuberculosis: a series of 15 cases. Clin Neurol Neurosurg 2009; 111: 115-8. 